Revista Brasileira de Farmacognosia Brazilian Journal of Pharmacognosy 21(5): 884-888, Sep./Oct. 2011

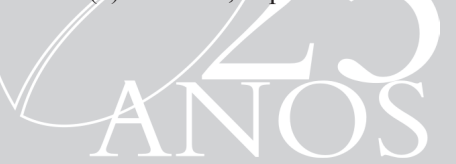

Article

Received 27 Dec 2010

Accepted 22 Mar 2011

Available online 17 Jun 2011

Keywords:

Wendtia calycina leaves

Phenylpropanoid glycosides

Human platelet aggregation

ISSN 0102-695X

http://dx.doi.org/10.1590/S0102-

$695 \times 2011005000109$

\section{Inhibition of human platelet aggregation in vitro by standardized extract of Wendtia calycina}

\author{
Milagros Garcia Mesa, ${ }^{1}$ Anna Lisa Piccinelli, ${ }^{2}$ María Antonia \\ Alfonso Valiente, ${ }^{1}$ Aldo Pinto, ${ }^{2}$ Alessia Fazio, ${ }^{3}$ Luca Rastrelli ${ }^{*}$,
}

\author{
${ }^{1}$ Departamento de Bioquímica, Instituto Nacional de Angiología y Cirugía \\ Vascular, Cuba, \\ ${ }^{2}$ Department of Pharmaceutical Sciences, School of Pharmacy, University \\ of Salerno, Italy, \\ ${ }^{3}$ Facoltà di Farmacia e di Scienze della Nutrizione e della Salute \\ dell'Università degli Studi della Calabria, Italy.
}

\begin{abstract}
Wendtia calycina (Griseb.) Griseb., Vivianiaceae, is a Paraguayan herbaceous plant commonly known as burrito. Our previous study indicated that burrito leaves are a very good source of phenylpropanoid glycosides, principally verbascoside. From W. calycina leaves, a standardized, water-soluble extract rich in phenylpropanoid glycosides (WSE) has been developed on an industrial scale to be used as a food supplement, cosmetic, phytomedicine, and ingredient of different formulations. In this study, we investigated the effect of the WSE on human platelet aggregation in vitro induced by adenosine diphosphate (ADP), epinephrine (EPN), collagen (COL) or arachidonic acid (AA). WSE, concentration-dependently, inhibited ADP and EP-induced human platelet aggregation (IC50 were $0.82 \pm 0.15$ $\mathrm{mg} / \mathrm{mL}$ and $0.41 \pm 0.02 \mathrm{mg} / \mathrm{mL}$, respectively). It did not inhibit collagen-induced platelet aggregation, thus suggesting a selectivity for the ADP-induced platelet activation pathways.
\end{abstract}

\section{Introduction}

Blood platelets are absolutely required for haemostatic plug formation when normal vessels are injured. However, the interactions between platelets and collagen can also cause circulatory disorders, such as thrombosis, atherosclerosis, and myocardial infarction (Schwartz et al., 1990). Atherothrombosis is one of the pathological events associated to oxidative reactions. Its most threatening consequences, myocardial infarction, stroke and lower-limb arterial thrombosis, are among the first causes of death and disability all over the World. The activation of blood platelets has a central role in atherothrombotic events. This activation is a consequence of platelet adhesion and aggregation on a damaged vascular endothelium giving the basis for the formation of arterial thrombus with the consequent blood flaw decrease and tissue ischemia.

An important role in the mechanism by which collagen induces platelet aggregation is played by thromboxane A2 (TXA2) formation (Cattaneo et al., 1991), which also contributes to an increase in cytosolic free $\mathrm{Ca}^{+2}$ level $\left(\left[\mathrm{Ca}^{+2}\right] \mathrm{i}\right)$ in collagen-activated platelets. An increase in $\left[\mathrm{Ca}^{+2}\right] \mathrm{i}$ activates both the $\mathrm{Ca}^{+2} /$ calmodulin- dependent phosphorylation of myosin light chain (20$\mathrm{kDa}$ ) and the diacylglycerol-dependent phosphorylation of cytosolic protein $(40-$ or $47-\mathrm{kDa})$ to induce platelet aggregation (Nishikawa et al., 1980; Kaibuchi et al., 1982) In addition, diacylglycerol also can be hydrolyzed by diacylglycerol lipase to produce arachidonic acid, a precursor of TXA2, which is a potent platelet aggregation agent generated from arachidonic acid liberated when PIP2 is broken down by stimulation with collagen, thrombin and ADP (Menshikov et al., 1993).

Inhibition of the platelet-collagen interaction might be a promising approach to the prevention of thrombosis. The mechanism of action of the antiplatelet drugs currently used in the medical practice are unrelated to the antioxidant action.

Theoreticaly, an antioxidant agent may inhibit platelet aggregation, since oxidative reactions are involved in platelet activation and aggregation (Maytin et al., 1999). However, recent studies have demonstrated that the antioxidant activity is involved in the mechanism of antiplatelet action of the flavonoids quercetin and catechin (Pignatelli et al. 2000; Singh et al., 2006). Epidemiological studies pointed out that diets rich in fruits and vegetables (rich in phenolic compounds) 
could prevent certain diseases in which free radicals are implicated. Since purified phenolic compounds are difficult to obtain and since extracts sometimes have biological activities higher than those of pure molecules, there is a growing interest for the use of plant extracts. Among them, Pycnogenol from French Pinus maritime bark, oligomeric proanthocyanidins from Vitis vinifera and Ginkgo biloba leaf extract are used in pharmacology and cosmetology or as a food supplement (Calliste et al., 2005).

Wendtia calycina (Griseb.) Griseb., Vivianiaceae, is a species that occurs mainly in Paraguay where is known as "burrito". The $W$. calycina leaves are used traditionally as a tea substitute, as well as a remedy to treat all kind of gastric disorders, inflammation and arthritis.

Our previous study indicated that burrito leaves are a very good source of phenylpropanoid glycosides, principally verbascoside and that its aqueous extract possessess in vitro antioxidant activity suggesting that it may be useful for the prevention of those pathologic events associated with oxidative stress (Piccinelli et al., 2004). From $W$. calycina leaves, a standardized, watersoluble extract (WSE) rich in phenylpropanoid glycosides has been developed on an industrial scale to be used as a food supplement, cosmetic, phytomedicine, and ingredient of different formulations. The extract is prepared by a standardized procedure that includes ultrasonic extraction with aqueous $\mathrm{EtOH}(60 \% \mathrm{v} / \mathrm{v})$, followed by a spraydrying using maltodextrin as excipient. WSE represents a concentrate of phenolic compounds and its main constituents are phenylpropanoid glycosides (PPGs), principally verbascoside $(7.5 \%)$ and isoverbascoside $(1.1 \%)$. This product also contains various benzoic acid derivatives as minor constituents. A recent study has shown the inhibition of inducible nitric oxide synthase in vitro and in vivo by water-soluble extract of $W$. calycina (WSE) (Marzocco et al., 2007). This work was aimed to assess the in vitro effect of WSE on human platelet aggregation induced by adenosine diphosphate (ADP), epinephrine $(\mathrm{EPN})$, collagen (COL) or arachidonic acid (AA).

\section{Materials and Methods}

\section{Plant material}

The leaves of Wendtia calycina (Griseb.) Griseb., Vivianiaceae, erroneously reported previously as Wendita calysina Martius (Piccinelli et al., 2004; Marzocco et al., 2007) were produced by Coprosa Ltd., Paraguay (Cooperativa multiactiva de Produccion Servicios publicos, Consumo, Ahorro y Credito, San Andres) and furnished by Commercio Altenativo S.r.l. (Ferrara, Italy), in February 2008. A specimen of the plant (No. K00053145) is deposited at the Herbarium Royal Botanic Gardens, Kew (K). The water soluble extract of $W$. calycina (WSE) was prepared by a standardized procedure that includes ultrasonic extraction with aqueous $\mathrm{EtOH} 60 \%$, followed by spray-drying using maltodextrins as excipient. Unless stated otherwise, all reagents and compounds used were obtained from Sigma Chemical Company (Sigma, Milan, Italy).

\section{Blood collection and platelet rich plasma preparation}

Blood of patients of both genders, aged from 19 to 55 years, participating in a routine health study, was obtained from Cerro Polyclinic in Havana City, Cuba. All enrolled subjects signed the informed consent and denied to have consumed any drug with known antiplatelet action at least two weeks before the flebotomy. Venous blood was obtained through plastic syringes containing $1 / 10(\mathrm{v} / \mathrm{v})$ of $3,8 \%(\mathrm{w} / \mathrm{v})$ trisodium citrate salt solution to avoid coagulation. Blood was then transferred in plastic tubes and platelet-rich plasma (PRP) was prepared by centrifugation at $150 \mathrm{x} g$ for $10 \mathrm{~min}$ at room temperature. After centrifugation the PRP was collected and the remaining blood was respun at $1000 \times \mathrm{g}$ for further 20 $\mathrm{min}$ at room temperature for preparation of platelet-poor plasma (PPP). A platelet count was performed on the PRP to ensure that the platelet concentration was in the range $220-250 \times 10^{3} / \mu \mathrm{L}$.

\section{Platelet aggregation}

W. calycina water-soluble extract (WSE) was diluted with a phosphate-buffered saline (PBS) solution at $\mathrm{pH} 7.4$ to assess its effect on platelet aggregation. A solution of acetylsalycilic acid (ASA) in 1\% sodium bicarbonate was used to assess the effect of the drug (5.5 $\mathrm{mmol} / \mathrm{L}$ in the incubation media) in the same conditions used for the WSE. Adenosine diphosphate (ADP), collagen, epinephrine, and arachidonic acid were obtained from CPM (CPM S.A.S, Rome, Italy) and prepared according to the instructions provided. The turbidimetric method (Born \& Cross, 1968) was applied to measure platelet aggregation, using a CLOT 2S Aggregometer (Seac and Radim Group, Rome, Italy). Five microliters of different dilutions of WSE in PBS (0.33, 0.66 and 1.32 $\mathrm{mg} / \mathrm{mL}$ ) or PBS (control) was added to $285 \mu \mathrm{L}$ aliquots of PRP in aggregometer cuvettes. Successively, $15 \mu \mathrm{L}$ of ADP, epinephrine, collagen, or arachidonic acid (final concentrations in the incubation media being $5 \mu \mathrm{mol} / \mathrm{L}$, $5 \mu \mathrm{mol} / \mathrm{L}, 3.2 \mu \mathrm{mol} / \mathrm{mL}$, and $0.6 \mathrm{mmol} / \mathrm{L}$, respectively) were added after 2 min of preincubation at $37^{\circ} \mathrm{C}$. Platelet aggregation was monitored for $5 \mathrm{~min}$. The results are expressed as percentages of aggregation as provided by the instrument. The percentage inhibition of platelet aggregation was calculated as follows: percentage inhibition (\%) [1 - (platelet aggregation of sample/ platelet aggregation of control) $] \times 100 \%$. Each sample was 
measured in triplicate. IC50 values (the concentration necessary to reduce the induced platelet aggregation by $50 \%$ with respect to control) were obtained from concentration-effect curves.

\section{Statistical analysis}

Data are expressed as percent of platelet aggregation. The value of WSE IC50 (the concentration necessary to reduce the platelet aggregation by $50 \%$ ) was obtained from concentration-effect curves of WSE. Data are expressed as the mean and the interval of confidence. The statistical analysis was performed by analysis of variance (ANOVA) by Kruskal-Wallis ranks and the Mann-Whitney U test. The maximum antiplatelet effect of WSE and ASA were compared by the Student's t test for independent data.

\section{Results and Discussion}

WSE in a concentration-dependent $(0.33,0.66$ or $1.32 \mathrm{mg} / \mathrm{mL}$ ) manner inhibited ADP-induced $(5 \mu \mathrm{mol} / \mathrm{L})$ human platelet aggregation (Figure 1). A statistically significant reduction, compared to vehicle-treated PRP, was observed to the concentrations of 0.66 and $1.32 \mathrm{mg} /$ $\mathrm{mL}(p<0.05$ and $p<0.001$ respectively). The calculated IC50 was $0.82 \pm 0.15 \mathrm{mg} / \mathrm{mL}$ (IC 0.52-1.11) (Table 1). In similar way, WSE treatment of human platelet in vitro produced aggregation reduction induced by EP ( 5 $\mu \mathrm{mol} / \mathrm{L}$ ) that was related in a concentration-dependent manner (Figure 2). Even though the WSE concentration of $0.33 \mathrm{mg} / \mathrm{mL}$ reduced of about $10 \%$ the platelet aggregation it was not statistically $(p>0.05)$ significant from control, whereas the concentration of 0.66 and 1.32 $\mathrm{mg} / \mathrm{mL}$ significantly inhibited human platelet aggregation $(p<0.05$ and $p<0.001$ respectively). The IC50 of $W$. calycina water-soluble extract in EP-induced aggregation was of $0.41 \pm 0.02 \mathrm{mg} / \mathrm{mL}$ in a range of IC $0.37-0.45 \mathrm{mg} /$ $\mathrm{mL}$. When the human platelet aggregation was induced by collagen $(2 \mu \mathrm{g} / \mathrm{mL})$ or AA $(0.5 \mathrm{mmol} / \mathrm{L})$ was not significantly impaired by the presence of WSE, at any tested concentrations (Figure 3 and 4 respectively).

Table 1. WSE concentration required to produced $50 \%$ inhibition (IC50) of human platelet aggregation induced by various aggregating agents.

\begin{tabular}{lccc}
\hline \multirow{2}{*}{ Aggregating agents (concentration) } & \multicolumn{3}{c}{$\mathrm{IC50}(\mathrm{mg} / \mathrm{mL})$} \\
\cline { 2 - 4 } & mean & sem & $\mathrm{cl}$ \\
\hline ADP $(5 \mu \mathrm{mol} / \mathrm{L})$ & 0.82 & 0.15 & $0.52-1.11$ \\
-Epinephrine $(5 \mu \mathrm{mol} / \mathrm{L})$ & 0.41 & 0.02 & $0.37-0.45$ \\
Collagen $(2 \mu \mathrm{g} / \mathrm{mL})$ & $>1.32^{\mathrm{a}}$ & - & - \\
Arachidonic acid $(0,5 \mathrm{mmol} / \mathrm{L})$ & $>1.32^{\mathrm{a}}$ & - & - \\
\hline
\end{tabular}

IC50 values were determined from each experiment $(n=9)$, through extrapolation from the curve percentage of inhibition versus log WSE concentration and expressed as mean + sem and confidence limit (cl) at $95 \%$.

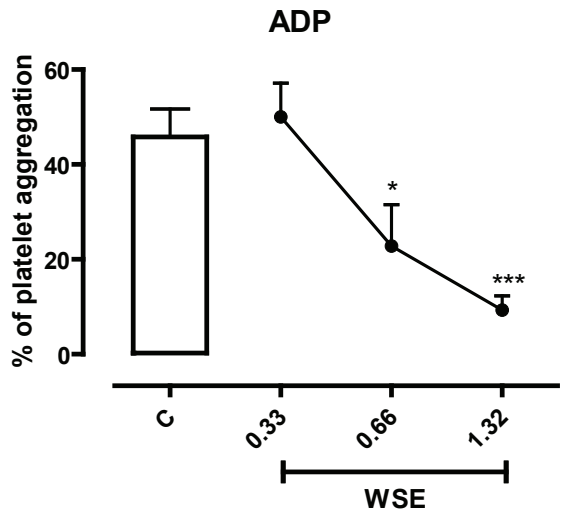

Figure 1. Effect of WSE on ADP ( $5 \mu \mathrm{mol} / \mathrm{L})$ induced aggregation. For platelet preparation see Material and Methods section. Results are expressed as mean+sem. $(n=9)$, and $* p<0.05, * * * p<0.001$.

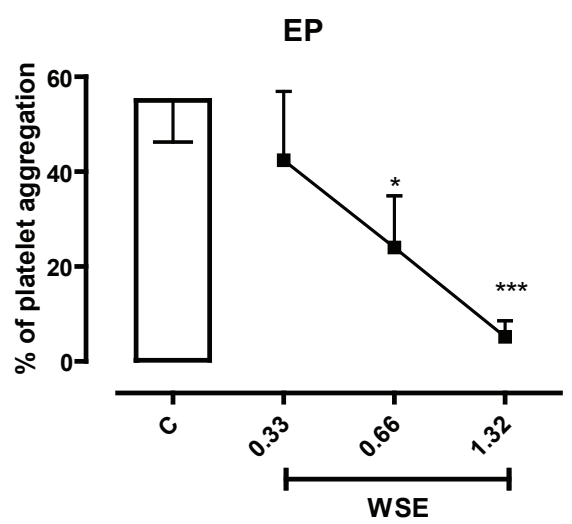

Figure 2. Effect of WSE on epinephrine (EP5 $\mu \mathrm{mol} / \mathrm{L})$ induced aggregation. For platelet preparation see Material and Methods section. Results are expressed as mean + sem. $(n=9)$ and $* p<0.05$, $* * * p<0.001$.

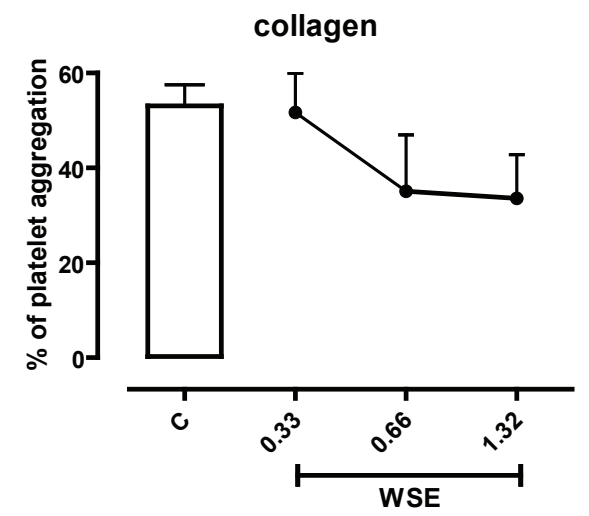

Figure 3. Effect of WSE on collagen $(3.2 \mu \mathrm{mol} / \mathrm{mL})$ induced aggregation. For platelet preparation see Material and Methods section. Results are expressed as mean $+\operatorname{sem}(n=9)$. 


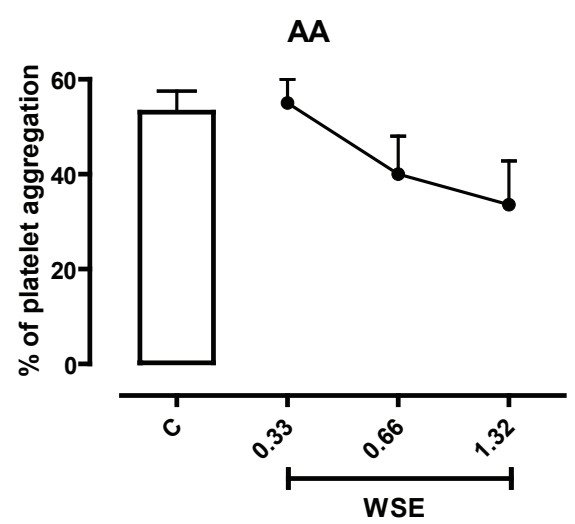

Figure 4. Effect of WSE on arachidonic acid (AA; 0.63 $\mu \mathrm{mol} / \mathrm{mL}$ ) induced aggregation. For platelet preparation see Material and Methods section. Results are expressed as mean + sem $(n=9)$.

The effect of ASA on human platelet aggregation induced by ADP, EP, collagen or AA was performed as reference drug. In our experimental model of human platelet aggregation, ASA $(5.5 \mathrm{mmol} / \mathrm{L})$ significantly $(p<0.001)$ inhibited the human platelet aggregation induced by collagen $(2 \mu \mathrm{g} / \mathrm{mL})$ and AA $(0.5 \mathrm{mmol} / \mathrm{L})$, but it was ineffective on the aggregation induced by ADP ( 5 $\mu \mathrm{mol} / \mathrm{L})$ or EP $(5 \mu \mathrm{mol} / \mathrm{L})$ (Figure 5$)$.

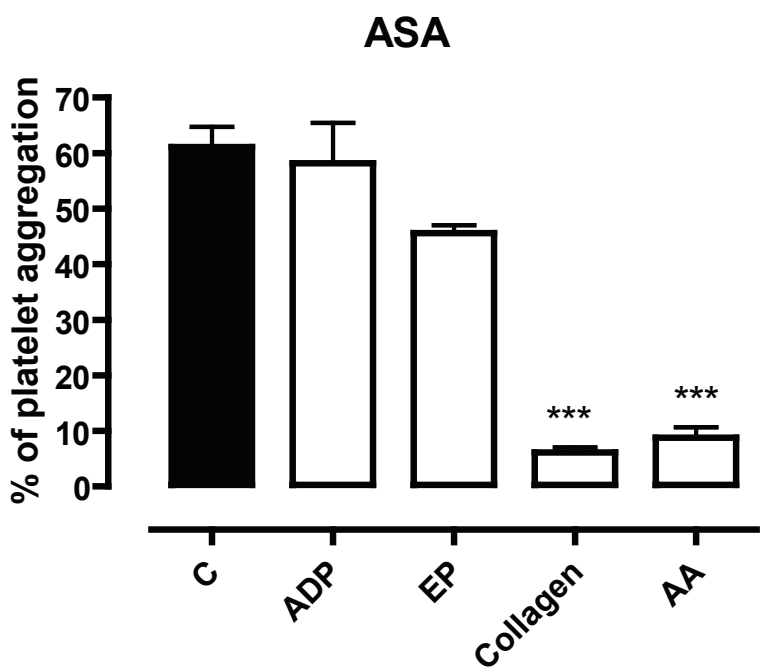

Figure 5. Effect of acetylsalycilic acid $(5.5 \mathrm{mmol} / \mathrm{L})$ on ADP $(5 \mu \mathrm{mol} / \mathrm{L})$, epinephrine $(\mathrm{EP} ; 5 \mu \mathrm{mol} / \mathrm{L})$, collagen $(3.2 \mu \mathrm{mol} /$ $\mathrm{mL})$, or arachidonic acid $(\mathrm{AA} ; 0.6 \mathrm{mmol} / \mathrm{L})$. For platelet preparation see Material and Methods section. Results are expressed as mean $+\operatorname{sem}(\mathrm{n}=4)$ and $* * * p<0.001$.

Different plant extracts have been used in the traditional medicines as remedies for various diseases. Generally, polyphenol-rich extracts have strong antioxidant activity, which can translate into several different beneficial human health effects. $W$. calycina leaves contain various phenolic compounds, including dihydroquercetin, methoxyflavones, methoxyflavonols, phenylpropanoid glycosides, and benzoic acid derivatives (Piccinelli et al., 2004).

These last two classes of compounds are watersoluble natural products and were found to be the main components of $W$. calycina water-soluble extract (WSE) particularly phenylpropanoid glycosides content (15.1 $\mathrm{mg} / 100 \mathrm{~mL}$ of aqueous infusion) appears to be very remarkable (Piccinelli et al., 2004). In this study, we have used ADP and collagen as aggregating agonists to assess the effect of WSE on human platelet aggregation in vitro. ADP and collagen have some differences as platelet aggregation stimuli. ADP stimulates blood platelets through the activation of specific purinergic receptors. GPVI is considered one of the glycoprotein which function as collagen receptors on platelets. The activation of these receptors is a signal for the increase of the cytoplasmic calcium, leading to platelet aggregation, thromboxane A2 formation and granules mediators liberation (known as the release reaction) such as ADP and serotonine, which amplify the response to the initial stimuli. Two aggregation waves may be clearly identified on ADP-aggregation curves: the first one (reversible) corresponds to the receptor activation signal and the second one (irreversible) corresponds to the effect of TXA2. On the other hand, collagen aggregation curves have a latency period, followed by a slow aggregate formation which depends on TXA2 formation. Therefore, agents which specifically block ADP receptors are good inhibitors of ADP- but weak inhibitors of collagen- induced platelet aggregation, this the case of the thienopyridin drugs, ticlopidine and clopidogrel. Aspirin-like drugs, which inhibit platelet cyclooxygenase and TXA2 formation, are good inhibitors of collagen-induced aggregation and inhibit the ADP second

WSE, concentration-dependently, inhibited ADP-induced platelet aggregation human PRP. It was an inhibitor as potent as ASA against ADP, however, It did not inhibit collagen-induced platelet aggregation, thus suggesting a selectivity for the ADP-induced platelet activation pathways.

The assayed WSE concentrations may be kind of high, however this not deny the importance of the pharmacological finding, since it is known that the degree of inhibition of platelet aggregation depends not only of the concentration of the antiplatelet agent, but also on the concentration of the aggregating agonist. We used the concentrations of ADP and collagen that produced submaximum aggregation in each plasma sample, consequently, platelet aggregation rates were always higher than $50 \%$ for both stimuli in control conditions. Besides, in our experimental conditions the ASA effective concentration against collagen was higher than expected according to the literature. Thus, 
it is possible that a effect of lower WSE concentration may be seen at milder conditions.

WSE is a $W$. calycina extract that have been prepared in similar way to that traditionally used in Paraguay. The extrapolation of in vitro to in vivo situations is quite difficult. However, our data suggest that those people who regularly consume this vegetable remedy may have a decreased platelet reactivity leading to a reduced susceptibility to atherothrombotic events. Future clinical assays will confirm or deny this hypothesis.

In conclusion, the results of this study confirm the hypothesis that a water-soluble extract of $W$. calycina has an antiplatelet action. This is the first experimental evidence of the antiplatelet potential of this plant. The future investigation will be directed to the characterization of this pharmacological action.

\section{References}

Born GVR, Cross MJJ 1968. The aggregation of blood platelets. J Physiol 108: 178-183.

Calliste CA, Trouillas P, Allai DP, Duroux JL 2005. Castanea sativa Mill. eaves as new sources of natural antioxidant: an electronic spin resonance study. $J$ Agric Food Chem 53: 282-288.

Cattaneo M, Tenconi PM, Lecchi A, Mannucci PM 1991. In vitro effects of picotamide on human platelet aggregation, the release reaction and thromboxane B2 production. Thromb Res 62: 717-724.

Kaibuchi K, Sano K, Hoshijima M, Takai Y, Nishizuka Y 1982. Phosphatidylinositol turnover in platelet activation; Calcium mobilization and protein phosphorylation. Cell Calcium 3: 323-335.

Maytin M, Leopold J, Loscalzo J 1999. Oxidant stress in the vasculature. Curr Atheroscler Rep 1:156-164.

Marzocco S, Piccinelli AL, Rastrelli L, Mazzon E, Cuzzocrea
S, Autore G 2007. Inhibition of inducible nitric oxide synthase in vitro and in vivo by a water-soluble extract of Wendtia calycina leave. Naunyn-Schmiedeberg's Arch Pharmacol 375: 349-358.

Menshikov MYu, Ivanova K, Schaefer M, Drummer C, Gerzer $\mathrm{R}$ 1993. Influence of the cGMP analog 8-PCPT-cGMP on agonist-induced increases in cytosolic ionized $\mathrm{Ca}^{2+}$ and on aggregation of human platelets. Eur $J$ Pharmacol 245: 281-284

Nishikawa M, Tanaka T, Hidaka H 1980. Calcium calmodulin dependent phosphorylation and platelet secretion. Nature 287: 863-865.

Piccinelli AL, De Simone F, Passi S, Rastrelli L 2004.) Phenolic constituents and antioxidant activity of Wendtia calycina leaves (burrito), a folk paraguayan tea. J Agric Food Chem 52: 5863-5868.

Pignatelli P, Pulcinelli FM, Celestini A, Lenti L, Ghiselli A, Gazzaniga PP, Violi F 2000. The flavonoids quercetin and catechin synergistically inhibit platelet function by antagonizing the intracellular production of hydrogen peroxide. Am J Clin Nutr 72: 1150-1155.

Schwartz SM, Heinmark RL, Majesky MW 1990. Developmental mechanisms underlying pathology of arteries. Physiol Rev 70: 1177-1209.

Singh I, Mok M, Christensen AM, Turner AH, Hawley JA 2008. The effects of polyphenols in olive leaves on platelet function. Nutr Metab Cardiovasc Dis 18: 127-132.

\section{*Correspondence}

Luca Rastrelli

Department of Pharmaceutical Sciences, School of Pharmacy, University of Salerno

Via Ponte don Melillo, 84084 Fisciano, Italy

rastrelli@unisa.it

Tel. +3989969766

Fax: +3989969602 